\title{
Task Priority Grasping and Locomotion Control of Modular Robot
}

\author{
G. Salvietti, H.X. Zhang, J. Gonzalez-Gòmez, D. Prattichizzo, J.W. Zhang
}

\begin{abstract}
This paper presents modular robots for grasping manipulation with locomotion capability. At the beginning, a brief overview on hyper-redundant and modular grasping approach is given. The innovations of this research lie in two points. Firstly, different grasping modes are integrated based on modular approach. Then manipulation capability of robotic arms and flexible locomotion mobility of mobile robots are combined in our current project. Furthermore, the approach in the paper is not only considering manipulation and mobility in modular robots but also, the exploitation of a task priority based approach introduced to manage the trade-off between these two functionalities. Related kinematics of our approach is present systematically. A rational simulation is also given to confirm the idea. In the end a conclusion is given and future work is outlined.
\end{abstract}

\section{INTRODUCTION}

A robot is an artificial, intelligent, autonomous system with a physical electro-mechanical platform which combines enough perception, manipulation capability or mobility to implement typical tasks. The research field of robotics has been contributing widely and significantly to industrial applications for assembly, welding, painting, and transportation for a long time. It is noted that manipulation and mobility are two important capabilities for robots. The former is necessary for robotic arms and industrial fixed manipulators; while the latter is the lowest basic functionality of the mobile robot systems for various applications.

The last few years have witnessed an increasing interest in integrating manipulation and mobility together for robots in order to enhance the flexibility, functionality and extensibility for current uses. An increasing number of possible applications encourage the researchers to concentrate on it, for example, searching and rescue tasks (SAR).

Urban search and rescue is a domain that involves a great amount of manpower; and it is quite dangerous and laborious in a hostile environment [1]. The development of mobile robots offers an intelligent alternative solution to the above-mentioned problems. The robotic system not only search, approach the victim but also provide some necessary treatment, even possible to grasp, rescue and carry the victim from the remains. The current solution is to integrate mobile

G. Salvietti is with Department of Information Engineering University of Siena, Via Roma 56, Siena, Italy salviettigio@dii.unisi.it

J. Gonzalez-Gòmez is with the School of Engineering, Universidad Autonoma de Madrid in Spain juan@iearobotics. com

H.X. Zhang is with the Institute of Technical Aspect of Multimodal System hzhangeinformatik. uni-hamburg. de

D. Prattichizzo is with Department of Information Engineering University of Siena prattichizzo@dii.unisi.it

J.W. Zhang is with the Institute of Technical Aspect of Multimodal System zhangeinformatik.uni-hamburg.de robot with wheels and powered trains with a simple manipulator, such as the HELIOS VIII robot designed S. Hirose [2], UMRS-NBCT robot [3] from international rescue robot institute. It usually happen that SAR working environment is very complicated, including not only high steps and deep ditches but also narrow fences and floors cluttered with debris. Normal wheeled and tracked mobile robots can not handle the situation easily according to the physic limitation. Therefore the robot should have the capability of adopting flexible linear configuration to match various tasks and suit complex environments.

Modular approach meets the requirements of flexibility, functionality and extensibility for future uses [4]. The possibility of grasp an object with snake-like configuration has been a critical need in SAR mission to enlarge passages around victims or while passing through an evadable but blocked area during exploratory navigation or transportation. Those capabilities can be used also to bring first aids or drugs to the trapped people.

This paper presents a novel modular grasping approach that combines the manipulation capability and locomotion mobility to implement possible tasks. The novelties of our approach lie in two aspects. Even if there are some similar grasping researches based on modular approach [5] [6], most of them are focused on different grasping kinematics and on control methods. Our research combines grasping manipulation in different modes and traveling mobility in the working scenario. Moreover a task priority based approach is introduced to manage grasping and locomotion function.

The paper is organized as follows: Section 2 introduce the related research work on modular robots and grasping manipulation. Then we will present our idea of combining the manipulation capability and mobility based on modular approach. In section 3, the locomotion and two possible grasping task oriented and motion oriented grasping ideas will be investigated throughout. The related kinematics will be presented to validate the principle. After that, in Section 4, the discussion focuses on the necessary simulation to confirm the integrated modular grasping and locomotion. In the end, future work and conclusions are given.

\section{RELATED WORK}

\section{A. Hyper-redundant grasping research}

There are two aspects related to our current modular grasping project, which are introduced in the following.

Grasping is a classical research topic in robotics [8], [9]. The interaction with the surrounding environment, consisting in the objects manipulation, is made by some living entities through the use of specific parts of the body, like hands for 


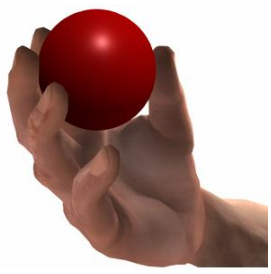

(a) Hand Grasping

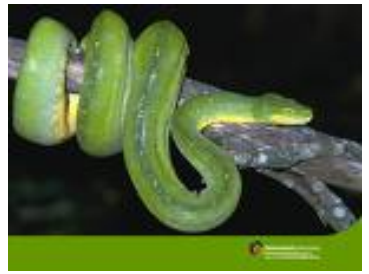

(b) Body grasping
Fig. 1. Two different grasping ways in nature

humans, and the whole body for some other like snakes. Hands and grippers can realize precise grasping while can even handle the object among the fingers, as shown in Fig. 1-a. A lot of research work has been done on hand grasping [10]. According to the difference of the grasping mechanisms, grasping can be for instance classified according to the number of fingers and contacts involved as twofingers, three-fingers and five-fingers grasping. More fingers involved, higher manipulability can be achieved. However, the controllability is getting worse with the increasing of the numbers of the fingers and contacts. Two fingers grasping is usually used as example for its simplicity. Since there are only two contact points between the manipulator and the object, the force control is relatively easy to be implemented. The disadvantage is that the grasping stability decreases and dexterous manipulation is not always achieved.

On the contrary side, the body grasping has an higher stability but worse manipulability. It is also called power grasping. The natural example of the snake's prey, is shown in Fig. 1-b. The whole body of the snake is involved in grasping so that there are many contact points between the manipulator and the object, thus improving the stability remarkably. Another advantage of body grasping in authors' opinion is that the flexibility of grasping is also improved. The grasping is not so sensitive to the dimension of the object. If the length of the manipulator body is longer than the biggest diameter of the object, the grasping is possible. From the research point of view, this grasping is suitable to dealing with unknown object. However, the increasing of the contact points number leads to a more difficult grasping control. Hyper redundant manipulator can be used to realize all these kinds of grasps.

The power grasping based on serial manipulators is capable of a wide variety of configurations to wrap its links around an object in the same manner used by octopus, tentacles and elephant trucks [11]. Some previous work has been done on this topic. In [5], authors investigated kinematics of modular hyper-redundant manipulators for manipulators with fixed based. In [12], authors studied a simple scheme for analyzing kinematics of hyper-redundant robot locomotion over solid terrain. The authors focused on the different locomotion gaits while also mentioned applications to grasp that was not completely analyzed. In a sentence, when the robot is grasping, it is a fixed manipulator, not a mobile robot at all. In a recent research the authors developed a distributed intelligent fuzzy learning controller for serpentine locomotion while doing lasso-type grasping in 3D environment [6]. They simulated a snake-like robot with 12 links to move and grasp the cylinder in the end. Actually, it is noted that the natural snake has an amazing locomotion capability in rugged terrains with different materials. But when the snake is holding something, it does not have the mobility since the whole body is involved into grasping manipulation to guarantee the grasping stability. The feasibility should be deduced clearly from the kinematics view point, which is not analyzed. Moreover, the lasso-type grasp involves a lot of links that cannot be used anymore for locomotion.

\section{B. Integrating different grasping modes and flexible mobility based on modular approach}

Modular robotic systems feature manifold functions, strong practicability, flexible expansibility and configuration, and robustness. They are usually composed of multiple building blocks of a relatively small repertoire, with uniform docking interfaces that allow transferring mechanical forces and moments, electrical power and communication throughout the whole robot [13].

Many research projects on modular robots were implemented worldwide [14], [15]. In 2004, our group began to work on low-cost passive modular robots. The Y1 modular robot with one DOF was designed to research the minimal configurations for movement [16]. Many locomotion capabilities including linear gait, lateral shifting, rotating gait, rolling gaits were simulated using Open Dynamic Engine (ODE) libraries [17] and on-site tested by an eight-module robot [18].

In this work, we will concentrate the efforts on combining various grasping including precise grasping and power grasping, and flexible mobility to enhance the flexibility, functionality, extensibility of modular robotic systems for possible tasks such as SAR. The modular approach enables robots to locomote and reconfigure flexibly, which is very essential for tasks which are difficult for a fixed-shape robot, thus also making the robotic system versatile, robust, and flexible to cross narrow passages.

Meanwhile, according to the target object's features, the modular robot can realize different grasps. It can grasp a cylinder only with two contacting points by reconfiguration, as shown in Fig. 2-a. While the other modules can still implement locomotion gaits. If necessary, the robot can increase the number of contact points in order to improve the stability, as shown in Fig. 2-b.

The following section of this paper will concentrate on the related kinematics and simulation to confirm our idea.

\section{MODULAR GRASPING}

\section{A. Task Priority Based Approach}

A task priority based approach is introduced to manage stability and manipulability in modular robot. The research of the best trade-off between these two capabilities is a classical problem when dealing with hyper-redundant mechanisms. In this work, a solution is proposed in a kinematic framework. 


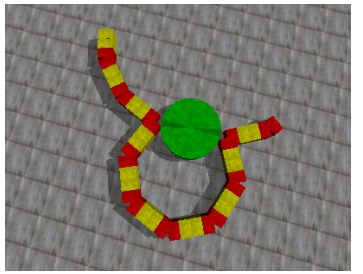

(a) Two fingers grasping

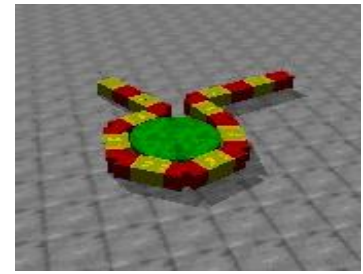

(b) Whole body grasping
Fig. 2. Various grasping modes with locomotion capabilities

During grasp operation our modular robot can be considered as a hyper-redundant manipulator. That is, matrix $J_{E}(q)$ in (1)

$$
\dot{x}_{E}=J_{E}(q) \dot{q}
$$

maps the $n$-dimensional space of joints velocities $\dot{q}$ in the $m$ dimensional space of end-effector velocities $\dot{x}_{E}$. In this case the last module involved in the grasp is considered to be end-effector, while the first is considered as reference point for the Jacobian matrix computing (Fig. 3).

If $n>m$, then the manipulator is kinematically redundant with respect to the given task and $(n-m)$ degrees-offreedom are available for solving redundancy. The joint velocity vector corresponding to a given end-effector task velocity vector can be found through the mapping (2)

$$
\dot{q}=J_{E}^{\dagger}(q) \dot{x}_{E}+N\left(J_{E}(q)\right) \dot{q}_{0}
$$

where ${ }^{\dagger}$ represents the Moore-Penrose pseudo-inverse and $N\left(J_{E}(q)\right)$ is a basis matrix of the nullspace of matrix $J_{E}(q)$, considering a non-minimum-norm solution based on the Jacobian pseudo-inverse. In a task priority approach, the choice of vector $\dot{q}_{0}$ can be obtained referring to a suitable constraint (3)

$$
\dot{x}_{C}=J_{C}(q) \dot{q}
$$

where the Jacobian matrix in (4)

$$
J_{C}=\delta x_{C} / \delta q
$$

is the $(m-n) \times n$ constraint task matrix. Equation (3) can be thought as a task with lower priority with respect to the endeffector task (1). A possible solution satisfying both tasks with their priorities is given in [20] and refer to (5)

$$
\dot{q}=J_{E}(q)^{\dagger} \dot{x}_{E}+\left(I-J_{E}(q)^{\dagger} J_{E}(q)\right) J_{C}(q)^{\dagger} \dot{x}_{C}
$$

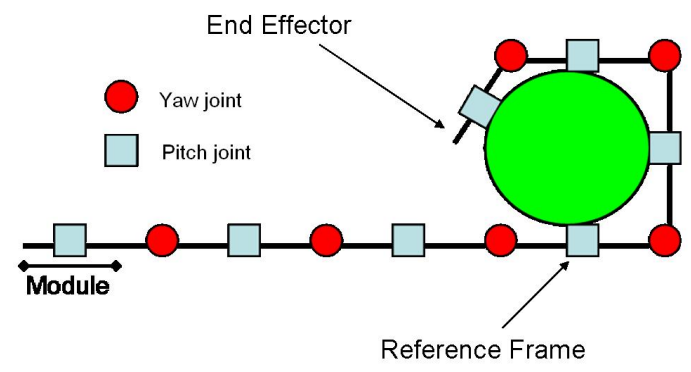

Fig. 3. End-effector and reference frame. where $\left(I-J_{E}(q)^{\dagger} J_{E}(q)\right)$ is the projector onto the nullspace of the first task. Using this method, the task with higher priority is always fulfilled, while the other is fulfilled only when it does not interfere with the higher one.

In this work we consider keeping a stable grasp as high priority task (HPT), while locomotion is the low-priority-task (LPT). We assume that the robot has already grasped the object and, moreover, that we are in quasi-static conditions, i.e. the inertia terms are negligible as occurs during slow motion.

For grasping analysis the modular robot can be assumed as a robotic hand, so that, HPT can be written in terms of the contact velocity constraint

$$
\left[\begin{array}{ll}
J(q) & -G(u)^{T}
\end{array}\right]\left[\begin{array}{c}
\dot{q} \\
\dot{u}
\end{array}\right]=0
$$

i.e. the velocities of contact points, thought as parts of the manipulator $J(q) \dot{q}$, and the velocities of contact points, thought as parts of the manipulated object $G(u)^{T} \dot{u}$, must be equal. $J(q)$ is the Hand Jacobian matrix, $G(u)$ is the grasp matrix as defined in [10] and $\dot{q}$ and $\dot{u}$ are the velocity of the modular robot and of the manipulated object, respectively.

According to [19], it can be proven that under quasi-static condition, the velocity $\dot{u}$ of the manipulated object can be written as a function of the joint velocity

$$
\dot{u}=\left(G(q) K G(q)^{T}\right)^{-1} G(q) K J(q) \dot{q}
$$

where $\mathrm{K}$ is the stiffness matrix at the contact points and can be considered equal to the identity matrix with the rigid body assumption. Combining the last two equations and considering grasping as the high priority task, HPT (1) can be written as

$$
J_{H}(q) \dot{q}=0
$$

where

$$
J_{H}=\left[J(q)-G(u)^{T}\left(G(u) G(u)^{T}\right)^{-1} G(u) J(q)\right]
$$

The fulfillment of the HPT is subject to the condition that the grasp is stable [10]. Note that the stability of the grasp can be obtained in many ways. One of these is the form closure condition: once a grasp configuration with relative contact points is defined, even if the robot moves, the grasp is maintained by geometrical constraints. Force closure grasps are more difficult to control with respect to the locomotion of the modular robot, but this is not covered in this work where a stable grasp during the motion of the robot is assumed. This assumption is less conservative for slow motion of the modular robot as in the case under investigation.

The LPT regards locomotion and ensure that the joints move with a suitable velocity that leads to a defined modular robot gait. The task can be written as in (10)

$$
\dot{x}_{L}=\dot{q}_{L}
$$

Substituting the task formulation in (5) and considering that $\dot{x}_{H}=0$ we obtain

$$
\dot{q}=M(q) \dot{x}_{L}
$$




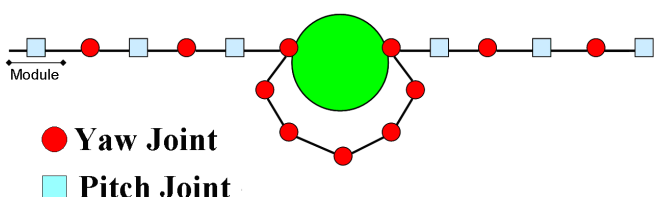

Pitch Joint

(a) Specific grasp section

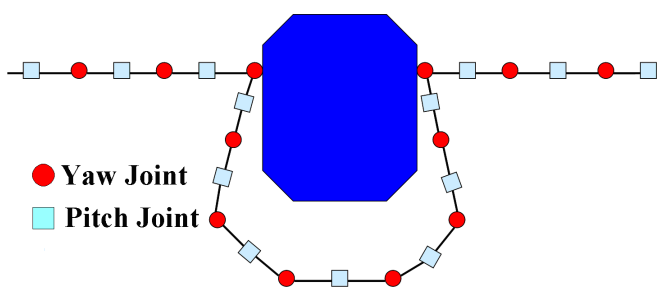

(b) Generic grasp section

Fig. 4. Two modular robot configurations: in the upper part, yaw joints functional to grasping are not able to move the modular robot. In the lower configuration, central section accounts for both locomotion and grasping tasks.

where

$$
M(q)=\left(I-J_{H}^{\dagger} J_{H}\right)
$$

The joint velocity to satisfy HPT and LPT is linear in the control variable for locomotion $\dot{x}_{L}$ that is imposed from the control system to the robot joints after a projection on the nullspace of the grasping high priority task. This projection allows to remove the components that could interfere with the grasping task. In other terms, the fulfillment of the high priority task is not affected by the lower one.

\section{B. Modular robot configuration}

Based on the modular idea it is possible to adapt the manipulator's shape to the object to grasp. We want to point out two different cases: a fixed number of module to use, and the case we can add as much module as needed. In the latter, modules can be added to the robot to reach the optimal shape to grasp and move objects. Currently with the GZ-I robot [7] it is possible to manage 256 modules. Different approaches are possible to establish the minimum number $n_{\min }$ of modules needed to accomplish grasping and locomotion tasks. For grasping we have used

$$
n_{\text {min }}=\frac{C_{o b j}}{l_{m}}
$$

where $C_{o b j}$ is the minimum length of a circle containing the object and $l_{m}$ is the length of one module.

Another important point is that we can consider sections of the robot devoted exclusively to grasping or locomotion tasks. In the upper part of Fig. 4 a modular robot with a specific grasping section is reported while in the lower part no special sections are considered and the whole modular robot parts can accomplish both grasping and locomotion tasks.

If the number of modules is given, the length of the grasp section is also affected by the type of gait we want to implement. If we want to move forward at least three modules

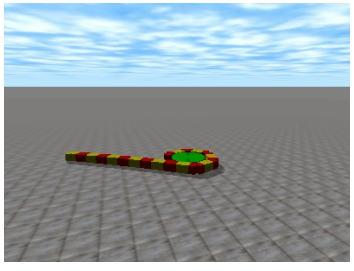

(a) Head grasping

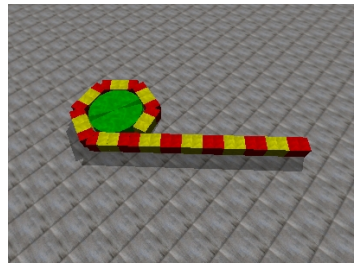

(b) Tail grasping
Fig. 5. Examples of head/tail grasp configurations.

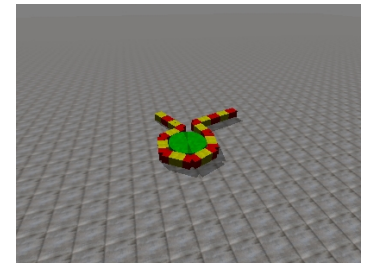

Fig. 6. Central body grasping.

are needed [22]. In a real application, the length of the locomotion section strictly depends on dynamic parameters. For instance, if an object is heavier than another with similar shape, we need more module in the locomotion section to move it.

We also want to increase the efficiency of locomotion in designing the modular robot with grasping and locomotion capabilities. This is not a problem because modular robots can be easily reconfigured by moving modules from one to another section. For instance, if the object has a particular shape which is difficult to grasp or in presence of rugged terrain during the locomotion, the grasping section can be powered by adding modules. If we want to increase the motion speed or, more generally, the locomotion capability of the robot, the locomotion section can be enlarged.

\section{Motion oriented grasping}

Together with the possibility of adapting the robot to the shape of the object to be grasped, we can also choose which is the most suitable configuration according to the direction of the motion. This solution can be useful in presence of obstacles in the workspace.

The capability of our robot to grasp objects using different parts of the body, increases the adaptability to critical situations. It is possible to define two macro motions oriented grasping

- tail/head grasping;

- central body grasping.

As shown in the figures, tail or head grasps are the most efficient when the modular robot moves in forward or backward (Fig. 5), while the central body grasp configuration exhibits better performances when we want to manipulate the object more than simply moving it (Fig. 6).

\section{GRASPING AND Locomotion Simulation}

To prove the reliability of the task priority approach several simulations have been performed using the Matlab 


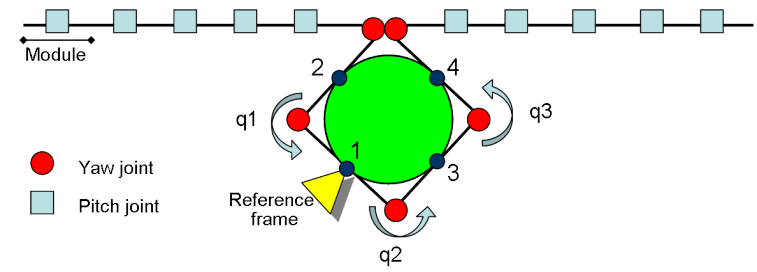

Fig. 7. Power grasp with a 15 modules robot

Robotics Toolbox [21] and ODE libraries.

Fig. 7 shows an example with 15-modules robot with the grasp section composed by yaw joints and the locomotion section by pitch joints only, where $q$ represent the joint's angles. We assume that the grasp is in a form closure condition and it is kept stable by geometrical constraint. Simulation has been updated with controlled kinematics as in (11) with

$$
\dot{x}_{L}=\left[\begin{array}{c}
A_{1} \cos \left(\frac{2 \pi}{T} t+\varphi_{1}\right) \frac{2 \pi}{T} \\
\vdots \\
A_{n} \cos \left(\frac{2 \pi}{T} t+\varphi_{n}\right) \frac{2 \pi}{T}
\end{array}\right]
$$

where $T=20, A_{n}=2 \sin \left(\frac{8 \pi}{n}\right)$ and $\varphi_{n}=\frac{8 \pi}{n}$ with n number of the modules. The initial joints angles are shown in (15)

$$
q(0)=\left[\begin{array}{llll}
\frac{3 \pi}{2} & \frac{3 \pi}{2} & \frac{\pi}{2} & 0 \ldots 0
\end{array}\right]
$$

Note that the given $\dot{x}_{L}$ has been chosen to perform a linear gait [22] and the initial joint angle to perform a form closure grasp.

Starting from a stable grasp condition, represented by the joint angle displacement vector $q(0)$, the next simulation step displacement can be computed as in (16)

$$
q(1)=q(0)+M(q(0)) \dot{q}_{L}(0) \Delta t .
$$

The yielding general law can be written as in (17)

$$
q(k+1)=q(k)+M(q(k)) \dot{q}_{L}(k) \Delta t .
$$

Fig. 8 represents the 15 -modules robot during the locomotion simulated with Matlab Robotics Toolbox. The blue cylinders are the joints and the yellow line the links. The grasp section is fixed and the other joints perform a linear gait moving forward. The joint angle displacement during the simulation is shown in Fig. 9.

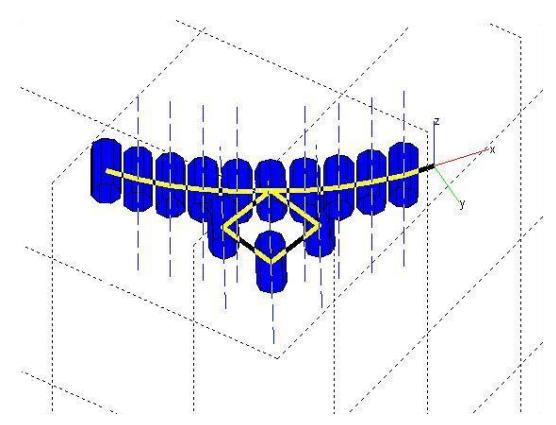

Fig. 8. The 15 module robot during grasp+locomotion

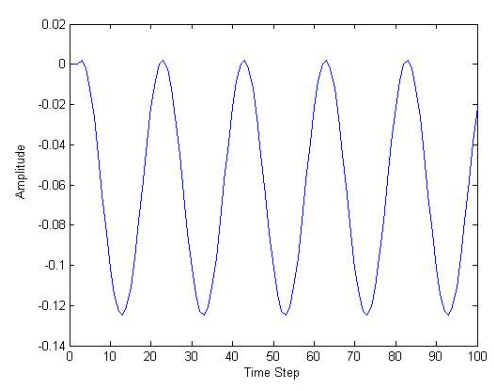

Fig. 9. The joint angle displacement during the simulation

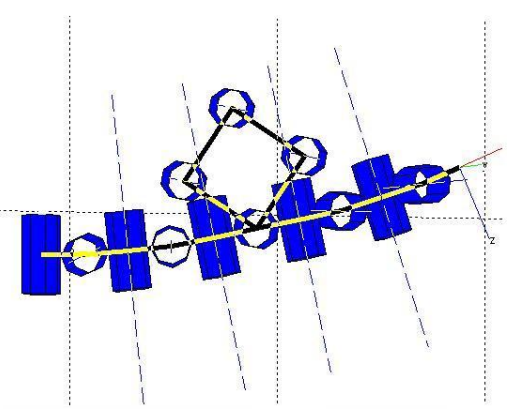

Fig. 10. The 19 module robot perform a lateral shift

Another configuration with 19-modules has been tested in order to reproduce more complicate gaits; e.g. in Fig. 10 a lateral shift is proposed. The stability of the grasp is guaranteed from the geometrical constraint and the pitch-yaw configuration is used to perform parallel movements. Other simulations have been done, using the ODE libraries, to show the capability of our robot to grasp and move an object. A possible implementation is now described with a 30-modules robot. The target object is the green cylinder in Fig. 11 and the yellow and red boxes represent respectively pitch and yaw joints. The snake uses different gaits to perform the approaching phase to the target.

First it moves in a straight line (Fig. 11-a) with an high amplitude to be fast. Then it changes to a flapping (Fig. 11b) gait towards the cylinder in order to obtain the better position for the grasp. When this is reached, the robot grasps the object (Fig. 11-c) performing a tail grasp. After that, the locomotion section start to move according to a linear gait while the locomotion section guarantee a force closure grasp (Fig. 11-d,11-e). For all the simulations we have supposed that the position of the object was known by the robot.

\section{CONCLUSIONS}

Modular reconfigurable robots have the ability of changing configurations which makes them more suitable for complex manipulation and locomotion in working everyday environments. The main contributions of this work are here summarized:

1) It proposes a new application of modular robots to integrate various grasping and locomotion capabilities based on reconfiguration concept. The advantages and 


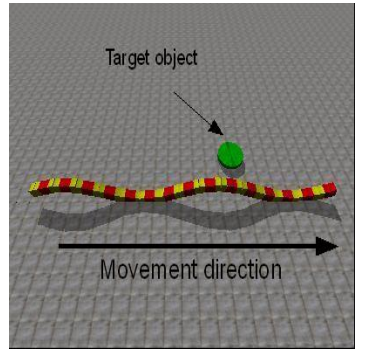

(a) Linear gait

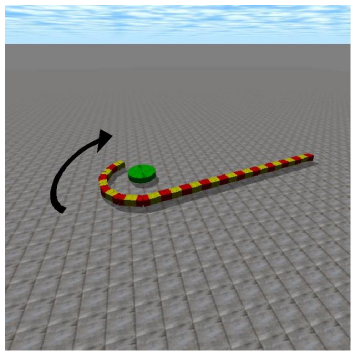

(c) Grasping

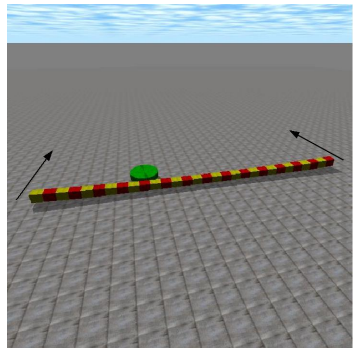

(b) Flapping

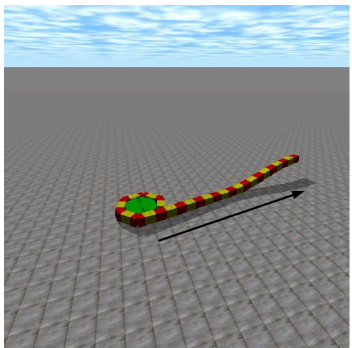

(d) Locomotion after grasping

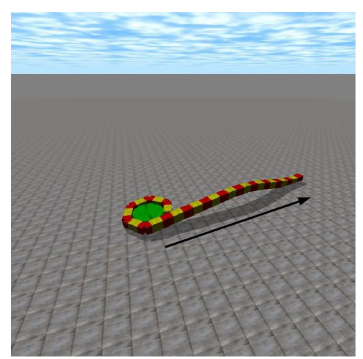

(e) Locomotion after grasping

Fig. 11. An example of tail grasping.

the characteristics of the modular grasping mechanism have been analyzed.

2) The related kinematic models of grasping have been investigated. The adaptability to different object to grasp shape and motion oriented grasping have been proposed.

3) The task priority based approach has been proposed as possible solution to manage different priorities in grasping and locomotion tasks.

4) Extensive simulations have been performed to validate the results of this work.

Currently, we are focusing the quantitative evaluation of the grasp and locomotion abilities of the modular robots and on the evaluation of the optimal number and relative orientation of the modules of the robot according to some performance index dealing with object manipulability and robot locomotion.

In addiction, experiments will be performed on a modular robot with all related problems dealing with sensing, actuation and computational power.

\section{ACKNOWLEDGMENTS}

This research is partially supported by exchanging master program between Italy and Germany.

\section{REFERENCES}

[1] F. Matsuno, S. Tadokoro, "Rescue Robots and Systems in Japan", Proceedings of Robio2004, Shenyang, China, 22-26 Aug. pp.12-20.

[2] M. Guarnieri, I. Takao, E. F. Fukushima, S. Hirose, Journal of Robotics and Mechatronics, Vol.20, No.5 pp. 675-694, 2008.

[3] Http://www.rescuesystem.org

[4] M. Yim, W. Shen, et al., "Modular Self-Reconfigurable Robot Systems: Challenges and Opportunities for the Future", IEEE Robotics and Automation Magazine, March 2007, pp.2-11.

[5] G.S. Chiriljian, J.W. Burdick, "Parallel Formulation of the Inverse Kinematics of Modular Hyper-Redundant Manipulators", Proceeding of the 1991 IEEE Robotics and Automation Conference, Sacramento, California, April, 1991, pp. 708-713.

[6] E. O. Ari, I. Erkmen, A. M. Erkmen, "FACL Based 3D Grasping Controller for a Snake Robot during Locomotion", Proceeding of the 2006 IEEE/RJS International Conference on Intelligent Robots and Systems, Oct.9-15, 2006, Beijing, China, pp. 4030-4035.

[7] H. Zhang, J. Gonzalez-Gomez, "Design and Development of Low-cost Modular robot GZ-I', Proceeding of AIM 2008, Xi'an, 2-5, July, China.

[8] M. Hüser, T. Baier-Löwenstein, J. Zhang, "Learning of demonstrated Grasping Skills by Stereoscopic Tracking of Human Hand Configuration", Proceeding of the 2006 IEEE International Conference on Robotics and Automation (ICRA), Orlando, Florida, USA, May 15-19, 2006, pp. 2795-2800.

[9] A. Bicchi, V. Kumar, "Robotic Grasping and Contact: A Review", Proceeding of the 2000 IEEE International Conference on Robotics and Automation(ICRA), San Francisco, CA, USA, 2000, pp. 348-353.

[10] D. Prattichizzo, J. C. Trinkle, "Grasping", Chapter 28th, Springer Handbook of Robotics, Springer 2008.

[11] J. S. Pettinato, H. S. Stephanou, "Manipulability and Stability of a Tentacle Based Robot Manipulator", Proceeding of the 1989 IEEE International Conference of Robot and Automation, may 15-19, Scottsdle, Az, USA, 1989, pp. 458-463.

[12] G. S. Chirikjian, J. W. Burdick, "Kinematics of Hyper-Redundant Robot Locomotion with Applications to Grasping”, Proceeding of the 1991 IEEE Robotics and Automation Conference, Sacramento, California, April, 1991, pp. 720-726

[13] Http://en.wikipedia.org/wiki/Self-Reconfiguring-Modular-Roboticsnote-space.

[14] M. Yim, Y. Zhang and E. Mao, "Distributed control for 3D shape Metamorphosis", Autonomous Robots, Vol.10, No.1, pp.41-56, 2001.

[15] S. Murata, E. Yoshida, A. Kamimura, H. Kurokawa, K. Tomita, S. Kokaji, "M-TRAN:Self-Reconfigurable Module Robotic System", IEEE/ASME Transactions on Mechatronics, Vol.7, No.4, pp.431-441, 2002.

[16] J. Gonzalez-Gomez, H. Zhang, E. Boemo, "Locomotion Principles of 1D Topology Pitch and Pitch-Yaw-Connecting Modular Robots", In: Bioinspiration and Robotics: Walking and Climbing Robots, Maki K. Habib (Ed.), pp.403-428. Advanced Robotic System and I-Tech.

[17] http://ode.org.

[18] J. Gonzalez-Gomez, H. Zhang, E. Boemo, J. Zhang, "Locomotion Capabilities of a Modular Robot with Eight Pitch-Yaw-Connecting Modules", Proceeding of CLAWAR 2006, Brussels, Belgium, September 12-14, 2006.

[19] A. Bicchi, "Force Distribution in Multiple Whole-Limb Manipulation", Robotics and Automation, Proceeding of the 1993 IEEE International Conference on Robotics and Automation, May 2-6 Atlanta, GA, USA, pp.196-201

[20] S. Chiaverini, "Singularity-Robust Task-Priority Redundancy Resolution for Real-Time Kinematics Control of Robot Manipulator", Robotics and Automation, Vol. 13, No. 3, 1997.

[21] P. I. Corke, "A Robotics Toolbox for MATLAB", IEEE Robotics and Automation Magazine, March 1996, pp 24-32.

[22] J. Gonzalez-Gomez, "Modular Robotics and Locomotion: Application to Limbless Robot”, Ph.D. Thesis, Madrid, December 2008. 